\title{
Analysis of KRAS, NRAS, BRAF, and PIK3CA mutations could predict metastases in colorectal cancer: A preliminary study
}

\author{
Kamila Wojas-Krawczyk ${ }^{1, A, C-F}$, Ewa Kalinka-Warzocha ${ }^{2, A, C-F}$, Katarzyna Reszka, ${ }^{3, B-D}$, Marcin Nicoś,B,C, \\ Justyna Szumiło ${ }^{4, B-D}$, Sławomir Mańdziuk ${ }^{5, B, C}$, Katarzyna Szczepaniak ${ }^{6, C, D}$, Dorota Kupnicka, ${ }^{7, B, C}$, \\ Remigiusz Lewandowski ${ }^{8, B, C}$, Janusz Milanowski ${ }^{1, A, D-F}$, Paweł Krawczyk ${ }^{1, A, D-F}$ \\ 1 Department of Pneumonology, Oncology and Allergology, Medical University of Lublin, Poland \\ ${ }^{2}$ Polish Mother's Memorial Hospital Research Institute, Łódź, Poland \\ ${ }^{3}$ Genetic and Immunology Institute GENIM Ltd., Lublin, Poland \\ ${ }^{4}$ Department of Clinical Patomorphology, Medical University of Lublin, Poland \\ ${ }^{5}$ Oncology and Chemotherapy Unit, Medical University of Lublin, Poland \\ ${ }^{6}$ Chemotherapy Department, Regional Centre of Oncology, Nicolaus Copernicus Provincial Specialist Hospital, Łódź, Poland \\ ${ }^{7}$ Pathology Laboratory Synevo, Łódź, Poland \\ ${ }^{8}$ MolGenDia Ltd., Bydgoszcz, Poland \\ A - research concept and design; $B$ - collection and/or assembly of data; $C$ - data analysis and interpretation; \\ $D$ - writing the article; $E$ - critical revision of the article; $F$ - final approval of the article
}

Address for correspondence

Kamila Wojas-Krawczyk

E-mail: kamilawojas@wp.pl

Funding sources

None declared

Conflict of interest

None declared

Received on January 4, 2017

Reviewed on January 20, 2017

Accepted on July 31, 2017

Published online on August 7, 2018

Cite as

Wojas-Krawczyk K, Kalinka-Warzocha E, Reszka K, et al. Analysis of $K R A S, N R A S, B R A F$, and PIK3CA mutations could predict metastases in colorectal cancer: A preliminary study. Adv Clin Exp Med. 2019;28(1):67-73. doi:10.17219/acem/76162

DOI

10.17219/acem/76162

Copyright

Copyright by Author(s)

This is an article distributed under the terms of the

Creative Commons Attribution Non-Commercial License

(http://creativecommons.org/licenses/by-nc-nd/4.0/)

\section{Abstract}

Background. Colorectal cancer (CRC) is usually diagnosed in the metastatic stage, when chemotherapy and molecularly-targeted therapies, instead of surgery, play the most important therapeutic role. Application of anti-epidermal growth factor receptor (EGFR) therapy requires the analysis of RAS mutation status and only RAS wild-type (wt) patients are qualified for the therapy.

Objectives. The objective of this study was to analyze driver mutations in KRAS, NRAS, BRAF, and PIK3CA genes in CRC patients.

Material and methods. We assessed the KRAS, NRAS, BRAF, and PIK3CA genes in 102 inoperable, locally advanced and advanced CRC patients. Real-time polymerase chain reaction (RT-PCR) and high resolution melt PCR (HRM-PCR) techniques with DNA intercalating dye were applied in the study.

Results. Forty-six patients demonstrated the presence of examined mutations (45.1\%). No significant differences in driver mutation occurrence between men and women, as well as between younger ( $<65$ years) and older ( $\geq 65$ years) patients were found. The mutations were present significantly more frequently in metastatic than in primary tumors $(p=0.039)$ due to the high incidence of KRAS gene mutations in metastatic tissue. BRAF and PIK3CA mutations were found only in primary tumors. The incidence of PIK3CA mutations was significantly higher (11.77\%) in early than in advanced stages of the disease $(1.96 \% ; p=0.05)$; NRAS mutations were found only in metastatic cancer $(7.85 \% ; p=0.041)$. Only a single mutation of the PIK3CA and no mutations of NRAS were found in rectal cancer.

Conclusions. Our results have shown low occurrence of driver mutations in Polish CRC patients, involving also mutations in rarely tested genes. The extent of the research panel of additional mutations could contribute to creating a better method of qualifying patients for molecularly targeted therapies and obtaining a better outcome for these therapeutic strategies.

Key words: colorectal cancer, PIK $3 C A, B R A F$, molecularly targeted therapy, RAS mutation 


\section{Introduction}

Colorectal cancer (CRC) is one of the leading causes of cancer death and one of the most common cancers among both men and women in developed countries. In Poland, approx. 16,000 new cases of CRC are registered every year; more than 10,000 people die (approx. 5,700 men and 4,800 women), and a 5-years overall survival rate among these patients is less than $50 \%$. The early diagnosis of cancers allows the patients to be qualified for surgery. Unfortunately, only small percentage of patients are qualified for surgical resection, and a significant proportion is diagnosed at a late stage, when, in the case of metastatic disease, surgical treatment is only a palliative procedure. Therefore, systemic treatment involving chemotherapy and molecularly targeted therapies plays an important role in the treatment of CRC. . $^{1-3}$

For several years, in many European countries, bevacizumab, cetuximab and panitumumab were the molecularly targeted drugs available for advanced CRC. ${ }^{3}$ The first one is a monoclonal antibody directed against vascular endothelial growth factor (VEGF), which inhibits angiogenesis and leads to the increase of blood vessel pressure. Two other drugs are monoclonal antibodies against epidermal growth factor receptor (EGFR), and their mechanism of action involves blocking the proliferation signal of tumor cells by inhibiting the EGFR signaling pathway (EGFR/ $\mathrm{Pi} 3 \mathrm{~K} / \mathrm{AKT} / \mathrm{mTOR}$ or EGFR/RAS/RAF/MAPK/ERK). ${ }^{3}$ Application of these drugs requires an analysis of $R A S$ gene mutations status and only patients with $R A S$ wildtype (wt) are qualified for the therapy. However, more than $40 \%$ of patients without common KRAS gene mutations (codon 12 and 13) do not respond to anti-EGFR monoclonal antibody therapies. This resistance can be explained by the presence of mutations in other downstream EGFR signaling pathway effectors, such as $B R A F, P I K 3 C A$ and $N R A S$ genes, as well as rare $K R A S$ gene mutations (codon $59,61,117,146) .^{2-4}$

In the present study, we assessed the occurrence of driver mutations in $K R A S$, NRAS, BRAF, and PIK3CA genes in inoperable, locally advanced and advanced CRC patients. We also estimated the association between the mutations incidence and clinical factors, especially tumor localization and stage of the disease.

\section{Material and methods}

The study group consisted of 102 CRC patients, including 69 (67.6\%) men and 33 (32.4\%) women, diagnosed in the Chemotherapy Department, Regional Centre of Oncology, Provincial Specialist Hospital in Łódź, Poland, and in the Oncology and Chemotherapy Unit of the Medical University of Lublin, Poland. The median age was $64 \pm 9.41$ years. All patients had advanced or inoperable mucinous adenocarcinoma CRC. Colorectal cancer grading and subtypes were not possible to assess in all patients due to the availability of only small biopsy specimens. Patients were qualified for anti-EGFR therapy with monoclonal antibodies (cetuximab or panitumumab monotherapy). For the purpose of the study, the following localizations of CRC adenocarcinoma were determined: 1. rectum; 2 . sigmoid colon; 3 . ascending, transverse and descending colon; and 4. CRC metastases. The clinical characteristics of patients are summarized in Table 1.

Archival tumor tissue from CRC as formalin-fixed paraffin embedded (FFPE) blocks was available from all patients enrolled in the study. The FFPE blocks were archived in the Department of Clinical Pathomorphology, Medical University of Lublin, Poland, and in Pathology Laboratory Synevo in Łódź, Poland. Mutation analysis was performed after a histological confirmation of cancer and when the presence of more than 10\% tumor cells was observed by a pathologist in hematoxylin and eosin (H\&E)-stained slides. Genomic DNA was extracted from the FFPE tumor tissue sections using a QIAamp DNA FFPE Tissue Kit (CE-IVD-marked; Qiagen, Hilden, Germany), according to the manufacturer's instructions. DNA concentration and quality was determined by the spectrophotometry method.

The study was approved by the Ethical Committee of the Medical University of Lublin, Poland (No. KE0254/218/2015). Informed consent was obtained from each participant.

The analysis of KRAS mutations was performed using $K R A S / B R A F$ Mutation Analysis Kit for real-time polymerase chain reaction (RT-PCR) (CE-IVD; EntroGen, Woodland Hills, USA) with a Cobas 480 RT-PCR device (Roche Diagnostics, Basel, Switzerland). The following mutations in the KRAS (NM_004985.3) gene, according to specific codon, were analyzed: codon 12 - p.Gly12Asp (c.35G>A), p.Gly12Cys (c.34G>T), p.Gly12Ser (c.34G>A), p.Gly12Arg (c.34G>C), p.Gly12Ala (c.35G>C), p.Gly12Val (c.35G>T); codon 13 - p.Gly13Asp (c.38G>A); codon 59 - p.Ala59Thr (c.174G>A), p.Ala59Glu (c.176C >A), p.Ala59Gly (c.176C>G); codon 61 - p.Gln61His (c.183A>C \& c.183A $>\mathrm{T}$ ), p.Gln61Leu (c.182A $>\mathrm{T})$, p.Gln61Arg (c.182A > G); codon 146 - p.Ala146X (c.436G>A, c.436G >C, or c.437C >T); codon 117 - p.Lys117X (c.351A >C, c.351A>T, c. $350 \mathrm{~A}>\mathrm{G}$, or c.349A $>\mathrm{G}$ ). The mutation analysis was carried out in relation to the amplification of positive and negative control tests provided by the manufacturer and according to the included protocol.

The analysis of the NRAS mutation was performed using the NRAS Mutation Analysis Kit for RT-PCR (CE-IVD; EntroGen) with a Cobas 480 RT-PCR device (Roche Diagnostics). The following mutations in NRAS (NM_002524.2) gene, according to codon, were analyzed: codon 12 - Gly12Asp (c.35G>A), Gly12Cys (c.34G>T), Gly12Ser (c.34G>A), Gly13Arg (c.37G>C); codon 13 - Gly13Val (c.38G $>$ T); codon 59 - Ala59Thr (c.175G>A), Ala59Asp (c.176C>A); codon 61 - Gln61His (c.183A>C \& c.183A>T), Gln61Leu (c.182A>T), 
Gln61Lys (c.181C>A), Gln61Arg (c.182A>G); codon 146 - Ala146Thr (c.436G >A); codon 117 - Lys117Arg (c.350A>G). The mutation analysis has been carried out in relation to the amplification of positive and negative control tests provided by the manufacturer and according to the included protocol.

The analysis of $B R A F$ mutations was performed using Cobas $^{\circledR} 480$ BRAF V600 Mutation Test (Roche Diagnostics). with a Cobas 480 RT-PCR device (Roche Diagnostics). The test detects Val600X (c.1799T>A or c.1798_1799GT>AA) mutations in the BRAF (NM_004333.4) gene and does not distinguish the type of mutations. The mutation analysis was carried out in relation to the amplification of positive and negative control tests provided by the manufacturer and according to the included protocol.

Estimation of the PIK3CA gene mutations (substitutions Glu542Lys, Glu545Lys, His1047Arg) was conducted using 2 methods based on a quantitative RT-PCR (qPCR). As a screening method, the high resolution melt PCR (HRM-PCR) technique was used. One pair of primers flanked the mutations located in exon 9 (substitutions Glu542Lys, c.1624G $>$ A and Glu545Lys, c.1633G $>A$ ), and the $2^{\text {nd }}$ pair of primers flanked a mutation located in exon 20 (substitution His1047Arg, c. $3140 \mathrm{~A}>\mathrm{G}$ ). The amplification of the examined region was performed using the Eco RT-PCR device (Illumina, San Diego, USA). Different genotypes of PIK3CA were distinguished according to the normalization data derived from the raw data plots and a difference graph derived from to the normalization data. Comparison of the amplification and melting curves in the positive and negative controls allowed us to distinguish the mutated-type (mt) and wt samples.

In the next step, the allele-specific qPCR (ASP-qPCR) method was used to define the type of mutations. The analysis was performed using the Eco RT-PCR device (Illumina). The wt and $\mathrm{mt}$ of the PIK3CA gene were tested in separate reactions with specific forward primers for these variants of the gene. Samples were assessed as positive if amplification in the ASPqPCR was observed both for the mt and for wt of the PIK3CA gene. The samples with late amplification ( $\mathrm{Ct}>32$ cycle)

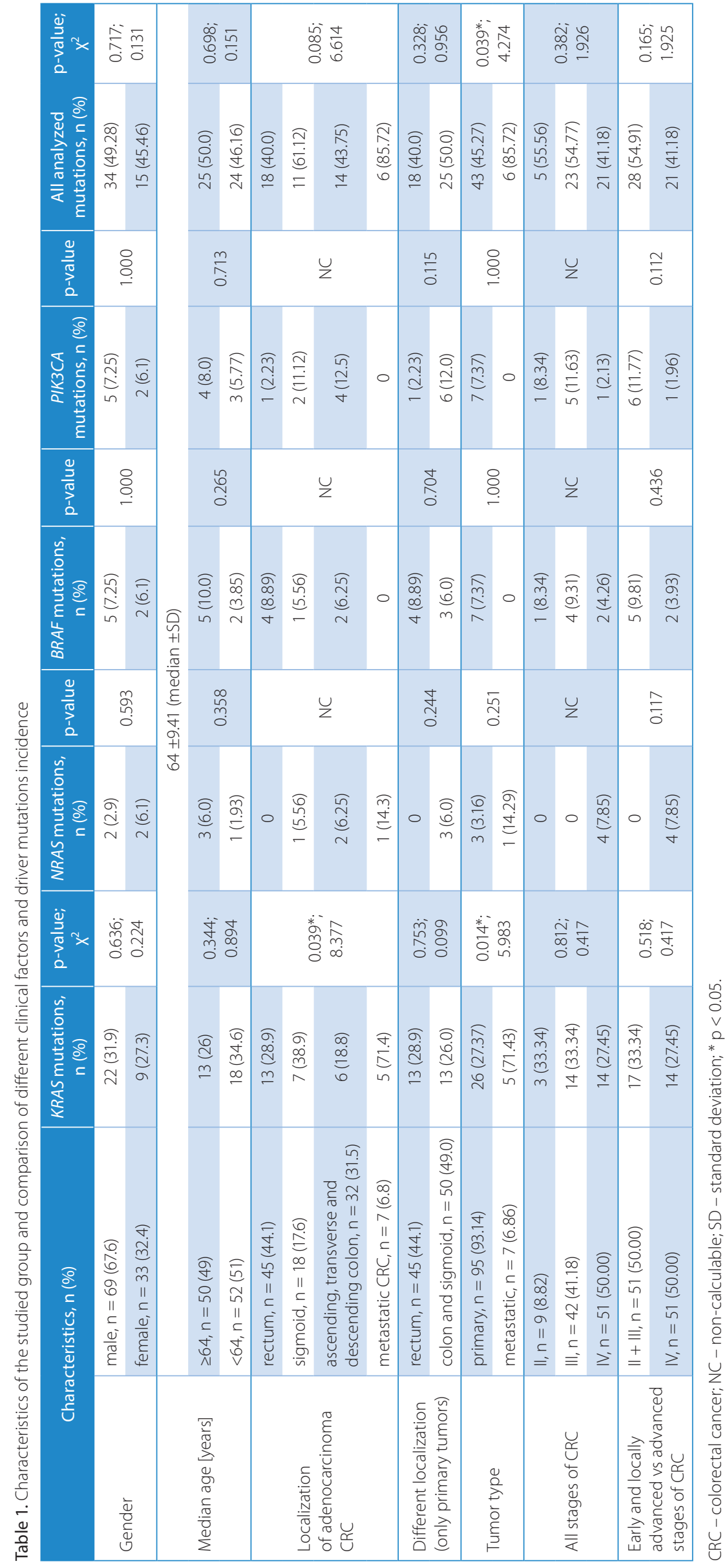


of the wt PIK3CA gene were excluded from analysis, and the samples with late amplification ( $\mathrm{Ct}>32$ cycle) of the $\mathrm{mt}$ region of the PIK3CA gene were assessed as wt. The representative analyses of mutations in PIK3CA genes analyzed by HRM-PCR and by ASP-qPCR were presented in Fig. 1 and 2 , respectively.

DNA isolated from the PIK3CA gene mutations positive cell-lines (SW48 cell-line for Glu542Lis; MCF10A cell-line for substitutions Glu545Lys and His1047Arg; Horizon Discovery, Cambridge, UK) served as a positive control. The negative control was determined with DNA isolated from the peripheral blood leukocytes of healthy individuals.

Statistical analysis was performed using STATISTICA, v. 10 (StatSoft Inc., Tulsa, USA). Associations between driver mutation occurrence and clinical factors were examined using Fisher's $x^{2}$ test if the expected values were greater than 5. Fisher's exact test was used if the expected values ranged from 0 to 4 . Student's t-test was used for testing the equality of population medians among groups (median age between groups); $\mathrm{p}$-values below 0.05 were considered significant.

\section{Results}

The median age was similar in the groups of patients with different clinical characteristics. Forty-six patients demonstrated the presence of any of the examined mutations (45.1\%). We found 53 driver mutations in the following genes: 35 mutations in KRAS gene, 4 mutations in NRAS gene, 7 mutations in BRAF gene, and 7 mutations in PIK3CA gene (Fig. 3).

In 2 cases, the coexistence of 3 of the examined mutations was found. KRAS codon 12 gene (Gly12Val) mutation coexisted with KRAS codon 117 (Lys117X) and PIK3CA codon 542 (Glu542Lys) mutations. In the $2^{\text {nd }}$ case, $K R A S$ codon 12 gene (Gly12Val) coexisted with KRAS codon 12 (Gly12Cys) and PIK3CA codon 1047 (His1047Arg) mutations. Moreover, in 3 cases we found the coexistence of 2 of the following mutations: KRAS codon 12 (Gly12Asp) mutation coexisted with KRAS codon 12 (Gly12Val) mutation, KRAS codon 12 (Gly12Cys) mutation coexisted with KRAS codon 146 (Ala146X) mutation, and KRAS codon 146 (Ala146X) mutation coexisted with PIK3CA codon 1047 (His1047Arg) mutation.

No differences in the incidence of driver mutations between men (49.28\%) and women (45.46\%) were found. The occurrence of mutations in KRAS, NRAS and $B R A F$ genes as well as in PIK3CA gene were very similar in men $(31.9 \%, 2.9 \%, 7.25 \%$, and $7.25 \%$, respectively) and in women $(27.3 \%, 6.1 \%, 6.1 \%$, and $6.1 \%$, respectively) (Table 1).

Depending on the age group, the incidence of KRAS mutations was insignificantly higher, while the incidence of NRAS, BRAF and PIK3CA gene mutations was

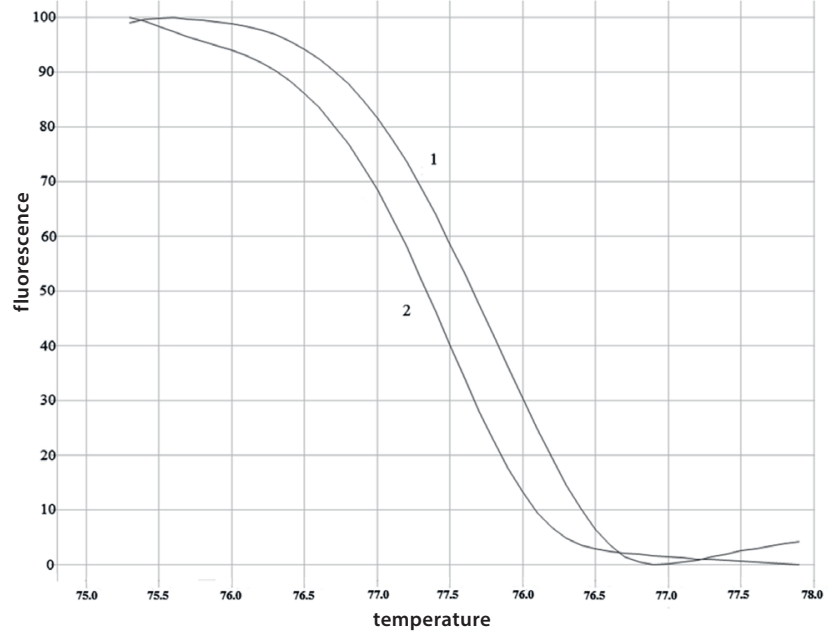

Fig. 1. Differences in melting curves of PIK3CA wt and mt genotypes. The H1047R mutation involves replacing of histidine (wt) by arginine (mt) in the 1047 position in the polypeptide chain. The mutation is caused by substitution of adeninie to guanine in the 3140 position of PIK3CA gene (exon 20). Comparison of melting temperatures and analysis of normalized data plots allowed distinguishing the wt and $\mathrm{mt}$ of PIK3CA gene

The melting curve 1 represents mt PIK3CA region, which corresponds to guanine in the position 3140 that needs higher temperature to melt the quantitative real-time polymerase chain reaction ( $\mathrm{PPCR}$ ) products; the melting curve 2 represents wt PIK3CA region, which corresponds to adenine in the position 3140 that needs lower temperature to melt the $\mathrm{qPCR}$ products.

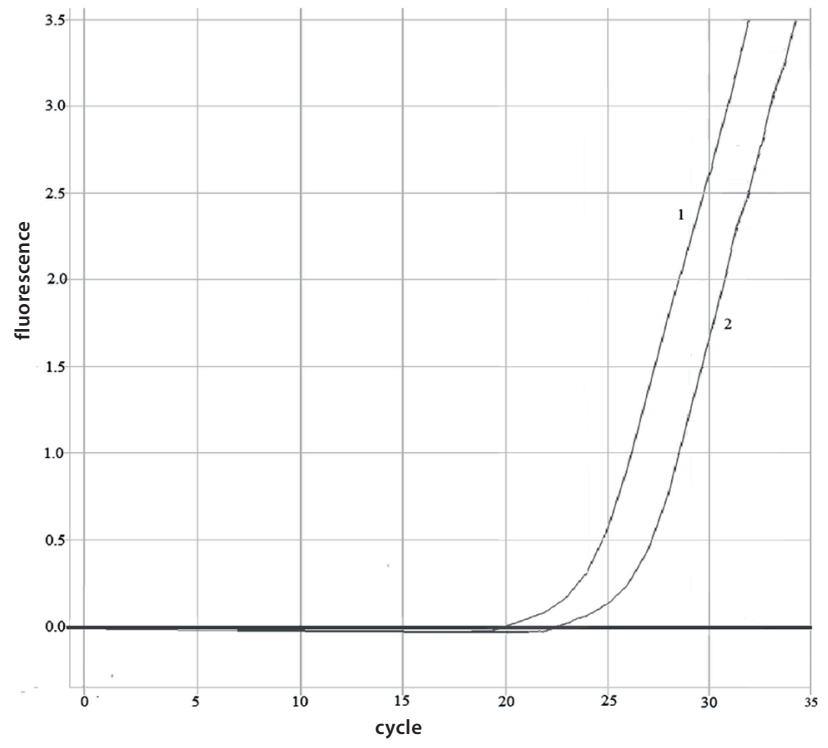

Fig. 2. Amplification curves of PIK3CA gene in allele-specific quantitative polymerase chain reaction (ASP-qPCR) analysis

Curve 1 represents amplification of wt PIK3CA region in negative control; curve 2 represents amplification of mt PIK3CA region in a positive patient.

insignificantly lower in younger patients, when compared to patients $\geq 64$ years old (Table 1 ).

There were no statistically significant differences in the incidence of analyzed mutations in rectal and colon cancer. However, only a single mutation of PIK3CA gene and no mutations of NRAS gene were found in rectal cancer. 


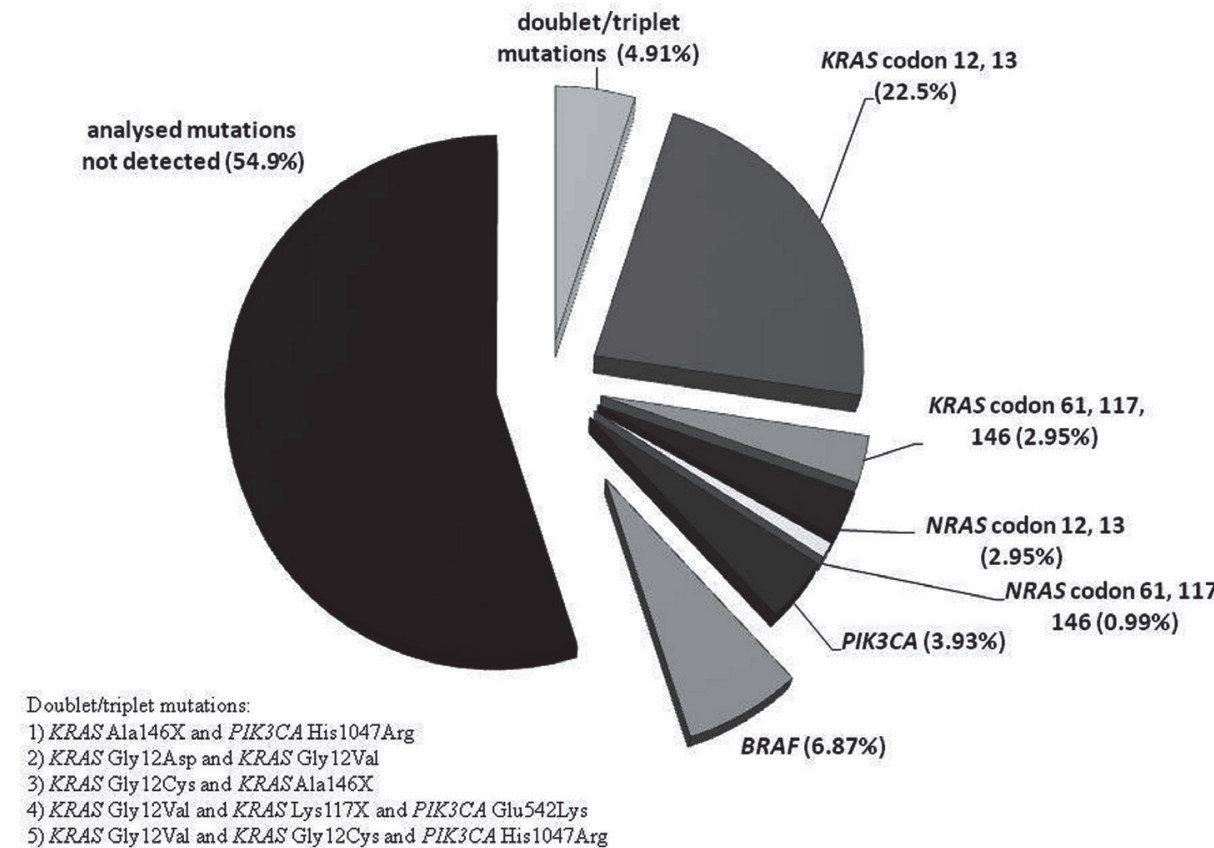

Fig. 3. The percentage of detected mutations in colorectal cancer (CRC) patients
The molecular profile of metastases seems to be different from the primary one. We found that the driver mutations were present significantly more frequently in metastatic tissue compared to primary tissue $\left(\mathrm{p}=0.039 ; \mathrm{x}^{2}=4.274\right)$. Specifically, a significantly higher incidence of $K R A S$ mutations was observed in metastatic tissue $(\mathrm{p}=0.014$; $\left.X^{2}=5.983\right)$ when compared to primary CRC (71.43\% vs $27.37 \%$, respectively); similar insignificant differences were observed for NRAS mutations (14.29\% vs 3.16\%). Moreover, $B R A F$ as well as PIK3CA mutations were observed only in primary tumor tissue (Table 1 ).

The incidence of the examined mutations was also analyzed according to CRC stages, and we found that it was similar for stages II and III of CRC (55.56\% and 54.77\%, respectively) and for the metastatic stage IV of CRC (41.18\%). When the earlier CRC stages were analyzed together, the incidence of PIK3CA mutations was insignificantly higher $(11.77 \%$; $\mathrm{p}=0.112)$ in stages II and III of CRC when compared to metastatic CRC (1.96\%). On the other hand, NRAS gene mutations were found only in the advanced stage of CRC (7.85\%; $\mathrm{p}=0.112$ ) (Table 1$)$.

\section{Discussion}

Nowadays, personalized cancer therapy rapidly becomes the standard care not only for CRC patients, but also in terms of therapies in other cancer patients. New molecular biology techniques are widely used for this purpose. ${ }^{3-5}$

De Sousa et al. distinguished 3 types of colon cancer subtypes using the whole-genome gene expression data and noticed that each type of cancer was associated with a different dominant feature and prognosis for the patient: CCS1 (epithelial type) and CCS2 (microsatellite instability - MSI type) were characterized by a rather good disease outcome, while CCS3 is of the mesenchymal dominant and had a bad prognosis. ${ }^{6}$ Nagtegaal and van Krieken proposed that CRC could be molecularly divided into 3 groups based on the epigenetic markers: chromosomal instability (CIN), MSI and CpG island methylation phenotype. ${ }^{4}$ It is also postulated that these types of tumors differ in terms of pathology, outcome and response to therapy, but this distinction is not used in clinical practice. ${ }^{4}$

From a practical point of view, it is difficult to require such wide molecular testing for CRC. In clinical practice, there are very few genetics laboratories where such broad molecular diagnostics could be performed. Therefore, we promote here an extended panel of $R A S$ gene mutations that could be helpful in a better qualification of CRC patients for molecularly targeted therapy. Furthermore, the use of diagnostic kits (CE-IVD-marked) for the determination of a wide panel of $R A S$ mutations should result in faster test release and a quicker decision in molecularly targeted therapy qualification. It could also help to understand the metastasis process and provide new prognostic factors in patients with CRC.

It is widely known that mutations in KRAS codons 12 and 13 predict the lack of response to anti-EGFR therapies. Vaughn et al. postulated that not only those mutations are responsible, but also additional, rarely tested activating mutations could play a role in treatment resistance. ${ }^{7}$ They found that $27.3 \%$ of tumors with KRAS wild-type codons 12 and 13 harbored mutation in codons 59, 61, 117, and 147 of the KRAS gene. In RAS wild-type CRC 
patients, PIK3CA mutations have been associated with a worse clinical outcome and with a negative prediction of response to targeted therapy with anti-EGFR monoclonal antibodies. ${ }^{7-9}$ Authors suggested that accurate testing of $R A S$ genes could be useful in identifying patients who may also be resistant to anti-EGFR therapies. ${ }^{7-9}$

In our examined population, we proved that driver mutations are present in relatively low numbers in CRC patients due to a low incidence of common KRAS mutations (codon 12 and 13). We analyzed 102 CRC patients and 46 (45.1\%) of them were found to carry 1 of the analyzed genes aberrations. Moreover, 5 patients were found to carry more than 1 mutation, including patients with coexistence of KRAS and PIK3CA genes mutations. This resulted in the inability to use monoclonal anti-EGFR therapy for those patients, but they had the possibility to be treated in specialized clinical trials.

In the Cetuximab Combined with Irinotecan in FirstLine Therapy for Metastatic Colorectal Cancer (CRYSTAL)study, a clinical benefit was observed for FOLinic acid-Fluorouracil-IRInotecan (FOLFIRI) scheme together with cetuximab addition in metastatic CRC patients with KRAS codon 12/13 wild-type. Moreover, the patients with the extended $R A S$ wild-type profile derived significant benefit from such therapy, while patients with any $R A S$ mutation did not. ${ }^{10,11}$ Based on the CRYSTAL study results, Van Cutsem et al. postulated that molecular testing for all activating $R A S$ mutations is essential for considering anti-EGFR therapy. ${ }^{12}$ Similar results were obtained in Oxaliplatin and Cetuximab in First-Line Treatment of Metastatic Colorectal Cancer (OPUS) (FOLFOX4 and cetuximab) as well as Panitumumab Efficacy in combination with mFOLFOX6 Against bevacizumab plus mFOLFOX6 in mCRC subjects with wild-type KRAS tumors (PEAK) (FOLFOX6 and panitumumab) and PRIME (FOLFOX and panitumumab) studies. ${ }^{13-15}$

There are only few reports in the literature concerning the differences between some genetic alterations and CRC localization. Slattery et al. analyzed the differences in the occurrence of driver mutations in colon and rectal tumors, and found that proximal colon cancers expressed more frequently microsatellite instability and had more $K R A S$ gene mutations than rectal and distal colon tumors, which are more likely to express $p 53$ gene mutations. ${ }^{16}$

We analyzed the incidence of driver mutations according to the localization of cancer as well as the type of tumor. When only primary tumors were considered, we found that KRAS driver mutations are more likely to localize in sigmoid colon cancer than in other tumor sites. Moreover, at this tumor location we observed the incidence of different mutations in any gene loci, including a rare mutation in $K R A S$ codon 146, and $N R A S$ codon 61 as well as the PIK3CA Glu542Lys mutations, which could indicate a preferential location of genetic changes at this site. Moreover, the predominant mutations found in rectal tumors were $K R A S$ codon 12 and BRAF Val600X. However, our work was carried out on a relatively small number of patients and confirmation of this thesis requires an extended study group. The coexistence of $K R A S$ with different other mutations could be related to the heterogeneity of the tumor and to the presence of these mutations in different clones of cancer cells. These clinical cases are especially difficult to treat with molecularly targeted therapy.

What is more interesting, we postulated that primary CRC seems to be different in terms of molecular profiling from metastatic tumors. Based on the very preliminary results, we tried to define the molecular profile of CRC metastases, which contain mutations in KRAS and NRAS genes, while they contain neither $B R A F$ nor $P I K 3 C A$ gene mutations. In contrast, the presence of $B R A F$ and PKI3CA mutations is characteristic for primary, early stages of CRCs.

Driver mutation status correlates not only with the response to anti-EGFR therapies, but also with metastatic spread. According to Lipsyc and Yaeger, the presence of $K R A S$ mutation is associated with an increased risk of lung, brain and bone metastases. BRAF and $K R A S$ genes mutations are rarely associated with the development of CRC liver metastases. BRAF mutation, a poor prognostic factor in metastatic CRC (mCRC), is associated with increased peritoneal and distant lymph node metastases. ${ }^{17}$ Moreover, the assessment of mutations in the RAS/BRAF and PIK3CA genes can not only be a predictor of the response to anti-EGFR antibody treatment, but these mutations could also help select patients who would achieve a better response to bevacizumab treatment. ${ }^{18}$ In the context of widening the routine molecular diagnostics, the meta-analysis conducted by Therkildsen et al. should be mentioned. ${ }^{19}$ The authors suggest that the biomarker analysis beyond KRAS exon 2 should be implemented for clinical benefit prediction from anti-EGFR antibodies in metastatic CRC. ${ }^{19}$

Summarizing, our preliminary study has shown a lower incidence of driver mutations in CRC patients, involving mutations not only in the widely studied KRAS codon 12 and 13, but also in rarely tested genes such as PIK3CA. Therefore, an in-depth molecular diagnosis seems to be necessary for proper qualifications to anti-EGFR therapy and for understanding the mechanisms of resistance to such therapies.

\section{References}

1. Akkad J, Bochum S, Martens UM. Personalized treatment for colorectal cancer: Novel developments and putative therapeutic strategies. Langenbecks Arch Surg. 2015;400(2):129-143.

2. Coppedè F, Lopomo A, Spisni R, Migliore L. Genetic and epigenetic biomarkers for diagnosis, prognosis and treatment of colorectal cancer. World J Gastroenterol. 2014;20(4):943-956.

3. Linnekamp JF, Wang X, Medema JP, Vermeulen L. Colorectal cancer heterogeneity and targeted therapy: A case for molecular disease subtypes. Cancer Res. 2015;75(2):245-249.

4. Nagtegaal ID, van Krieken JH. Colorectal cancer: Is the new era of colorectal cancer classification finally here? Nat Rev Gastroenterol Hepatol. 2013;10(7):391-393. 
5. Kim TM, Lee SH, Chung YJ. Clinical applications of next-generation sequencing in colorectal cancers. World J Gastroenterol. 2013;19(40):6784-6793.

6. De Sousa E, Melo F, Wang X, et al. Poor-prognosis colon cance is defined by a molecularly distinct subtype and develops from serrated precursor lesions. Nat Med. 2013;19(5):614-618.

7. Vaughn CP, Zobell SD, Furtado LV, Baker CL, Samowittz WS. Frequency of KRAS, NRAS and BRAF mutations in colorectal cancer. Genes Chromosomes Cancer. 2011;50(5):307-312.

8. Cathomas G. PIK3CA in colorectal cancer. Front Oncol. 2014;4:35. doi: 10.3389/fonc. 2014.00035

9. Sartore-Bianchi A, Martini M, Veronese FMS, et al. PIK3CA mutations in colorectal cancer are associated with clinical resistance to EGFRtargeted monoclonal antibodies. Cancer Res. 2009;69(5):1851-1857.

10. De Roock W, Claes B, Bernasconi $\mathrm{D}$, et al. Effects of $K R A S, B R A F, N R A S$ and PIK3CA mutations on the efficacy of cetuximab plus chemotherapy in chemotherapy-refractory metastatic colorectal cancer: A retrospective consortium analysis. Lancet Oncol. 2010;11(8):753-762.

11. Loupakis F, Ruzzo A, Cremolini C, et al. KRAS codon 61, 146 and BRAF mutations predict resistance to cetuximab plus irinotecan in KRAS codon 12 and 13 wild-type metastatic colorectal cancer. Br J Cancer. 2009;101(4):715-721.

12. Van Cutsem $\mathrm{E}$, Lenz HJ, Köhne $\mathrm{CH}$, et al. Fluorouracil, leucovorin, and irinotecan plus cetuximab treatment and RAS mutations in colorectal cancer. J Clin Oncol. 2015;33(7):692-700.
13. Bokemeyer $\mathrm{C}$, Kohne $\mathrm{CH}$, Ciardiello $\mathrm{F}$, et al. Treatment outcome according to tumour RAS mutation status in OPUS study patients with metastatic colorectal cancer (mCRC) randomized to FOLFOX4 with/without cetuximab. J Clin Oncol. 2014;32(15 Suppl):abstr 3505.

14. Oliner K, Douillard J, Siena S, et al. Analysis of KRAS/NRAS and BRAF mutations in the phase III PRIME study of panitumumab and FOLFOX vs FOLFOX as first line treatment for metastatic colorectal cancer. J Clin Oncol. 2013;31(15 Suppl):abstr 3511

15. Schwartzberg LS, Rivera F, Karthaus M, et al. PEAK: A randomized, multi-centre phase II study of panitumumab plus modified fluorouracil, leucovorin, and oxaliplatin (mFOLFOX6) or bevacizumab plus mFOLFOX6 in patients with previously untreated, unresectable, wild-type KRAS exon 2 metastatic colorectal cancer. J Clin Oncol. 2014;32(21):2240-2247.

16. Slattery ML, Curtin K, Wolff RK, et al. A comparison of colon and rectal somatic DNA alterations. Dis Colon Rectum. 2009;52(7):1304-1311.

17. Lipsyc M, Yaeger R. Impact of somatic mutations on patterns of metastasis in colorectal cancer. J Gastrointest Oncol. 2015;6(6):645-649.

18. Nakayama I, Shinozaki E, Matsushima T, et al. Retrospective study of RAS/PIK3CA/BRAF tumor mutations as predictors of response to first-line chemotherapy with bevacizumab in metastatic colorectal cancer patients. BMC. 2017;17(1):38-46.

19. Therkildsen $C$, Bergmann TK, Henrichsen-Schnack T, Ladelund $S$, Nilbert M. The predictive value of KRAS, NRAS, BRAF, PIK3CA and PTEN for anti-EGFR treatment in metastatic colorectal cancer: A systemic review and meta-analysis. Acta Oncol. 2014;53(7):852-864. 